\title{
ON COMPACT EINSTEIN DOUBLY WARPED PRODUCT MANIFOLDS
}

\author{
PUNAM GUPTA
}

\begin{abstract}
In this paper, the non-existence of connected, compact Einstein doubly warped product semi-Riemannian manifold with non-positive scalar curvature is proved. It is also shown that there does not exist non-trivial connected Einstein doubly warped product semi-Riemannian manifold with compact base $B$ or fibre $F$.
\end{abstract}

\section{Introduction}

In 1969, Bishop and O’ Neill [6] introduced singly warped products or warped products to construct Riemannian manifolds with negative sectional curvature. Warped products have significant applications in many areas like general relativity, solutions of Einstein's equations [1],[4] and in many areas of geometry because of their role in construction of new examples with interesting curvature and symmetry properties [5, 8, 11, 23].

After that, theory of relativity demands a larger class of manifold and then idea of doubly warped products was introduced. In 1983, O’Neill [23] discussed doubly warped products and derived curvature formulas of doubly warped products in terms of curvatures of components of doubly warped products. Doubly warped products and its properties are studied by many authors [12, 13, 14, 21, 24, 26, 27] in different aspects.

In 1983, Besse [5, p.265] gave the open problem "Does there exist a compact Einstein warped product space with nonconstant warping function?" In 2002, Kim [16] studied the Einstein warped product spaces and proved that there does not exist a compact Einstein warped product space if the scalar curvature is non-positive or the base is of 2-dimensional. In 2003, Kim and Kim [17] gave the partial answer of Besse question and showed that there does not exist an Einstein warped product space with nonconstant warping function if the scalar curvature is non-positive and base is compact. In 2005, Mustafa [22] generalized the result of Kim and Kim [17] and proved the same result with no condition on scalar curvature. For semi-Riemannian manifold, Beem et al. [4] and Easely [10] proved that if $M=B \times_{f} F$ is a 4dimensional Ricci flat warped product, where base $B$ as a surface of constant curvature, then

Received September 28, 2017, accepted May 9, 2018.

2010 Mathematics Subject Classification. 53C05, 53C25, 53C50.

Key words and phrases. Doubly warped product manifold, compact manifold, Einstein manifold. 
$M$ is simply a product manifold. Compact Einstein warped product Riemannian manifold has been studied by many authors $[7,9,18]$.

The purpose of this paper is to extend the result of $[16,17,22]$. The non-existence of connected compact Einstein doubly warped product semi-Riemannian manifold with nonconstant warping function is proved if the scalar curvature is non-positive. In the last, it is shown that there does not exist non-trivial connected Einstein doubly warped product semiRiemannian manifold with compact base $B$ or compact fibre $F$. After that, an open problem in the Finslerian set up is given.

\section{Preliminary}

Doubly warped product manifolds were introduced as a generalization of warped product manifolds. Let $\left(B, g_{B}\right)$ and $\left(F, g_{F}\right)$ be two semi-Riemannian manifolds with real dimension $n_{1}$ and $n_{2}$, respectively. Let $h: B \rightarrow \mathbb{R}^{+}$and $f: F \rightarrow \mathbb{R}^{+}$be two smooth functions. Consider the product manifold $B \times F$ with its projections $\pi: B \times F \rightarrow B$ and $\sigma: B \times F \rightarrow F$. The doubly warped product ${ }_{h} B \times{ }_{f} F$ is the product manifold $B \times F$ furnished with the metric tensor

$$
g=(f \circ \sigma)^{2} \pi^{*}\left(g_{B}\right)+(h \circ \pi)^{2} \sigma^{*}\left(g_{F}\right),
$$

where $^{*}$ denotes pullback. If $X$ is tangent to $B \times F$ at $(p, q)$, then

$$
g(X, X)=f^{2}(q) g_{B}(d \pi(X), d \pi(X))+h^{2}(p) g_{F}(d \sigma(X), d \sigma(X)) .
$$

Thus we have

$$
g=f^{2} g_{B}+h^{2} g_{F}
$$

The function $h$ and $f$ are called the warping functions of the doubly warped product. The manifold $B$ is known as the base of $(M, g)$ and the manifold $F$ is known as the fibre of $(M, g)$. If the warping function $f$ or $h$ is constant, then the doubly warped product ${ }_{h} B \times{ }_{f} F$ reduces to a warped product ${ }_{h} B \times \tilde{F}$ (or $\tilde{B} \times{ }_{f} F$ ), where the fibre $\tilde{F}$ is just $F$ with metric $\tilde{g}_{F}$ given by $\frac{1}{h^{2}} g_{F}$ (or where the base $\tilde{B}$ is just $B$ with metric $\tilde{g}_{B}$ given by $\frac{1}{f^{2}} g_{B}$ ). If the warping function $f$ or $h$ is equal to 1 , then the doubly warped product ${ }_{h} B \times{ }_{f} F$ reduces to a warped product ${ }_{h} B \times F$ (or $B \times{ }_{f} F$ ). If both $f$ and $h$ are constant, then it is simply a product manifold $\tilde{B} \times \tilde{F}$, where the fibre $\tilde{F}$ is just $F$ with metric $\tilde{g}_{F}$ given by $\frac{1}{h^{2}} g_{F}$ and the base $\tilde{B}$ is just $B$ with metric $\tilde{g}_{B}$ given by $\frac{1}{f^{2}} g_{B}$. If both $f$ and $h$ are equal to 1 , then it is simply a product manifold $B \times F$.

The set of all smooth and positive valued functions $h: B \rightarrow \mathbb{R}^{+}$and $f: F \rightarrow \mathbb{R}^{+}$are denoted by $\mathscr{F}(B)=C^{\infty}(B)$ and $\mathscr{F}(F)=C^{\infty}(F)$, respectively. The lift of $h$ and $f$ to $M$ are defined by $\tilde{h}=$ $h \circ \pi \in \mathscr{F}(M)$ and $\tilde{f}=f \circ \sigma \in \mathscr{F}(M)$. If $X_{p} \in T_{p}(B)$ and $q \in F$, then the lift $\tilde{X}_{(p, q)}$ of $X_{p}$ to $M$ is the unique tangent vector in $T_{(p, q)}(B \times\{q\})$ such that $d \pi_{(p, q)}\left(\tilde{X}_{(p, q)}\right)=X_{p}$ and $d \sigma_{(p, q)}\left(\tilde{X}_{(p, q)}\right)=0$. 
The set of all such horizontal tangent vector lifts will be denoted by $L_{(p, q)}(B)$. Similarly, we can define the set of all vertical tangent vector lifts $L_{(p, q)}(F)$.

Let $X \in \mathfrak{X}(B)$, where $\mathfrak{X}(B)$ is the set of smooth vector fields, then the lift $\tilde{X}$ of $X$ to $M$ is the unique element of $\mathfrak{X}(M)$ whose value at each $(p, q)$ is the lift of $X_{p}$ to $(p, q)$. The set of such lifts will be denoted by $\mathscr{L}(B)$. In a similar manner, we can define $\mathscr{L}(F)$. Throughout the paper, we assume that $X, Y, Z \in \mathscr{L}(B)$ and $U, V, W \in \mathscr{L}(F)$. The connections $\nabla,{ }^{B} \nabla$ and ${ }^{F} \nabla$ are Levi-Civita connections on $M, B$ and $F$, respectively. The tensors $S,{ }^{B} S$ and ${ }^{F} S$ are the Ricci tensors for the connections $\nabla,{ }^{B} \nabla$ and ${ }^{F} \nabla$, respectively; $r,{ }^{B} r$ and ${ }^{F} r$ are the scalar curvatures for the connections $\nabla,{ }^{B} \nabla$ and ${ }^{F} \nabla$, respectively.

A semi-Riemannian manifold $M$ is said to be an Einstein manifold [23] if its Ricci tensor $S$ is proportional to the semi-Riemannian metric $g$, i.e. $S=\mu g$ for some $\mu \in C^{\infty}(M)$. If $\operatorname{dim} M>$ 2 and $M$ is connected, then $\mu$ is constant.

Now, we define

Definition 2.1. An Einstein doubly warped product semi-Riemannian manifold (Einstein DWP semi-Riemannian manifold) is a doubly warped product semi-Riemannian manifold which is Einstein.

\section{Doubly warped product semi-Riemannian manifold}

In this section, we give some results on Einstein doubly warped product semi-Riemannian manifolds.

Lemma 3.1 ([15]). Let $M={ }_{h} B \times{ }_{f} F$ be a doubly warped product semi-Riemannian manifold. Then

$$
\begin{aligned}
S(X, Y)= & { }^{B} S(X, Y)-\frac{n_{2}}{h} H_{B}^{h}(X, Y) \\
& -\frac{1}{f^{2}}\left(\left(n_{1}-1\right) g_{F}(\nabla f, \nabla f)+f \Delta_{F} f\right) g(X, Y),
\end{aligned}
$$

where $H_{B}^{h}$ is the Hessian of $h$ on $B$ and $\Delta_{F} f$ is the Laplacian of $f$ on $F$.

$$
\begin{aligned}
S(X, V)= & \frac{(n-2)(X h)(V f)}{h f}, \\
S(V, W)= & { }^{F} S(V, W)-\frac{n_{1}}{f} H_{F}^{f}(V, W) \\
& -\frac{1}{h^{2}}\left(\left(n_{2}-1\right) g_{B}(\nabla h, \nabla h)+h \Delta_{B} h\right) g(V, W),
\end{aligned}
$$

where $H_{F}^{f}$ is the Hessian of $f$ on F.and $\Delta_{B} h$ is the Laplacian of $h$ on $B$. 
By using the above Lemma, we have the following result.

Corollary 3.2. Let $M={ }_{h} B \times{ }_{f} F$ be a connected doubly warped product semi-Riemannian manifold. Then $M$ is Einstein manifold with Einstein constant $\mu$ if and only if

$$
\begin{aligned}
{ }^{B} S(X, Y) & =\lambda_{1} g_{B}(X, Y)+\frac{n_{2}}{h} H_{B}^{h}(X, Y), \\
\lambda_{1} & =\left(\left(n_{1}-1\right) g_{F}(\nabla f, \nabla f)+f \Delta_{F} f+f^{2} \mu\right), \\
{ }^{F} S(V, W) & =\lambda_{2} g_{F}(V, W)+\frac{n_{1}}{f} H_{F}^{f}(V, W), \\
\lambda_{2} & =\left(\left(n_{2}-1\right) g_{B}(\nabla h, \nabla h)+h \Delta_{B} h+h^{2} \mu\right) .
\end{aligned}
$$

Remark 3.3. Above Corollary 3.2, gives existence of Einstein DWP semi-Riemannian manifolds.

Lemma 3.4 ([17]). Let $\varphi$ be a smooth function on a Riemannian manifold $M$. Then for any vector field $X$ in $M$, the divergence of the Hessian tensor $H^{\varphi}$ satisfies

$$
\operatorname{div}\left(H^{\varphi}\right)(X)=S(\nabla \varphi, X)-\triangle(d \varphi)(X)
$$

where $\triangle$ denotes the Laplacian on $M$.

Note: This Lemma is true for semi-Riemannian manifold case also.

Proposition 3.5. Let $\left(B, g_{B}\right)$ be a connected compact semi-Riemannian manifold with $n_{1} \geq 2$ and $h$ be a non-constant smooth function on $B$ satisfying (3.4) for $\lambda_{1} \in \mathbb{R}$ and $n_{2} \in \mathbb{N}$. Then $h$ satisfies (3.7) for $\lambda_{2} \in \mathbb{R}$.

Proof. On contraction of (3.4) with respect to $X$ and $Y$, we have

$$
{ }^{B} r=\lambda_{1} n_{1}-\frac{n_{2}}{h} \triangle_{B} h
$$

By using Bianchi second identity, we can easily obtain

$$
\nabla_{X}^{B} r=2 \operatorname{div}\left({ }^{B} Q X\right)
$$

From (3.9) and (3.10), we get

$$
\operatorname{div}\left({ }^{B} Q X\right)=\frac{n_{2}}{2 h^{2}}\left\{\left(\Delta_{B} h\right) d h-h d\left(\Delta_{B} h\right)\right\} X .
$$

It is easy to show that

$$
\operatorname{div}\left(\frac{H^{h}}{h}\right)(X)=-\frac{1}{h^{2}} H^{h}(\nabla h, X)+\frac{1}{h} \operatorname{div} H^{h}(X) .
$$


Let $\left\{e_{1}, \cdots, e_{n_{1}}\right\}$ be an orthonormal frame of $B$. Then

$$
\begin{aligned}
H^{h}(X, \nabla h) & =\frac{1}{2} d\left(|\nabla h|^{2}\right)(X), \\
\operatorname{div}\left(\frac{H^{h}}{h}\right) & =-\frac{1}{2 h^{2}} d\left(|\nabla h|^{2}\right)+\frac{1}{h} \operatorname{div} H^{h} .
\end{aligned}
$$

Using (3.4), (3.8) and (3.13) in (3.14), we obtain

$$
\operatorname{div}\left(\frac{H^{h}}{h}\right)=\frac{1}{2 h^{2}}\left(\left(n_{2}-1\right) d\left(|\nabla h|^{2}\right)-2 h \triangle(d h)+2 \lambda_{1} h d h\right) .
$$

By (3.4), we have

$$
\operatorname{div}\left({ }^{B} S(X, Y)\right)=\operatorname{div}\left(\frac{n_{2}}{h} H_{B}^{h}(X, Y)\right) .
$$

Therefore by (3.11) and (3.15), we obtain $0=d\left(\left(n_{2}-1\right) g_{B}(\nabla h, \nabla h)+h \Delta_{B} h+h^{2} \mu\right)$ and hence (3.7).

Similarly, we have the result.

Proposition 3.6. Let $\left(F, g_{F}\right)$ be a connected compact semi-Riemannian manifold with $n_{2} \geq 2$ and $f$ be a non-constant smooth function on $F$ satisfying (3.6) for $\lambda_{2} \in \mathbb{R}$ and $n_{1} \in \mathbb{N}$. Then $f$ satisfies (3.5) for $\lambda_{1} \in \mathbb{R}$.

Hence by using Propositions 3.5 and 3.6, we can form a connected compact Einstein DWP semi-Riemannian manifold $M={ }_{h} B \times{ }_{f} F$.

The above necessary condition yields the following non-existence result for connected compact Einstein DWP semi-Riemannian manifold with non-positive scalar curvature.

Theorem 3.7. Let $M={ }_{h} B \times{ }_{f} F$ be a connected, compact Einstein DWP semi-Riemannian manifold with non-positive scalar curvature. Then doubly warped product manifold is simply warped product or product semi-Riemannian manifold.

Proof. By (3.5), we get

$$
\lambda_{1}=\left(n_{1}-2\right) g_{F}(\nabla f, \nabla f)+\operatorname{div}(f \nabla f)+f^{2} \mu .
$$

On integration (3.17) over $F$, we have

$$
\lambda_{1}=\frac{\left(n_{1}-2\right)}{V(F)} \int_{F} g_{F}(\nabla f, \nabla f)+\frac{\mu}{V(F)} \int_{F} f^{2},
$$

where $V(F)$ denotes the volume of $F$. There are two cases: 
Case I. Let $n_{1}>2$. Take $p$ as a maximum point of $f$ on $F$, then $f(p)>0, \nabla f(p)=0$ and $\triangle f(p) \leq 0$, therefore

$$
f(p) \triangle f(p) \leq 0
$$

Using (3.17), we have

$$
\lambda_{1}-\mu f(p)^{2} \leq 0
$$

By (3.18), we get

$$
\frac{\left(n_{1}-2\right)}{V(F)} \int_{F} g_{F}(\nabla f, \nabla f)+\frac{\mu}{V(F)} \int_{F}\left(f^{2}-f(p)^{2}\right) \leq 0,
$$

but the above quantity is greater than or equal to zero, by virtue of our hypothesis because we take non-positive scalar curvature. Therefore, we can say that $f$ is constant.

Case II. Let $n_{1}=2$. Take $q$ a minimum point of $f$ on $F$, then $f(q)>0, \nabla f(q)=0$ and $\triangle f(q) \geq 0$, therefore

$$
f(q) \triangle f(q) \geq 0
$$

Using (3.5), we have

$$
\lambda_{1}-\mu f(q)^{2} \geq 0
$$

By (3.18), we get

$$
\frac{\left(n_{1}-2\right)}{V(F)} \int_{F} g_{F}(\nabla f, \nabla f)+\frac{\mu}{V(F)} \int_{F}\left(f^{2}-f(q)^{2}\right) \geq 0
$$

but the above quantity is less than or equal to zero, by same reason, which shows that $f$ is constant.

In the similar process by using (3.7), we can show that $h$ is constant. This completes the proof.

Proposition 3.8. Let $M={ }_{h} B \times{ }_{f} F$ be a connected Einstein DWP semi-Riemannian manifold with Einstein constant $\mu,\left(B, g_{B}\right)$ and $\left(F, g_{F}\right)$ be compact semi-Riemannian manifolds. If either

1. Either $r^{B} \geq \mu n_{1}+\frac{n_{1}\left(n_{1}-1\right)}{f^{2}} g_{F}(\nabla f, \nabla f)$ or $r^{B} \leq \mu n_{1}+\frac{n_{1}\left(n_{1}-1\right)}{f^{2}} g_{F}(\nabla f, \nabla f)$, or,

2. Either $r^{F} \geq \mu n_{2}+\frac{n_{2}\left(n_{2}-1\right)}{h^{2}} g_{B}(\nabla h, \nabla h)$ or $r^{F} \leq \mu n_{2}+\frac{n_{2}\left(n_{2}-1\right)}{h^{2}} g_{B}(\nabla h, \nabla h)$ holds.

Then the warping functions $h$ and $f$ are constants and, up to a scale, $M$ is a simply product semi-Riemannian manifold.

Proof. By (3.1), we have

$$
\sum_{i=1}^{n_{1}} S\left(e_{i}, e_{i}\right)=r^{B}-\frac{n_{2}}{h} \Delta_{B} h-\frac{1}{f^{2}}\left(\left(n_{1}-1\right) g_{F}(\nabla f, \nabla f)+f \Delta_{F} f\right) n_{1} .
$$


Given that $M$ is Einstein manifold with Einstein constant $\mu$, therefore the above equation reduces to

$$
\mu n_{1}-r^{B}+\frac{n_{1}\left(n_{1}-1\right)}{f^{2}} g_{F}(\nabla f, \nabla f)=-\frac{n_{2}}{h} \Delta_{B} h-\frac{n_{1}}{f} \Delta_{F} f .
$$

Similarly, by (3.3) and using the fact that $M$ is Einstein manifold with Einstein constant $\mu$, we have

$$
\mu n_{2}-r^{F}+\frac{n_{2}\left(n_{2}-1\right)}{h^{2}} g_{B}(\nabla h, \nabla h)=-\frac{n_{2}}{h} \Delta_{B} h-\frac{n_{1}}{f} \Delta_{F} f
$$

Now, if

1) either $r^{B} \geq \mu n_{1}+\frac{n_{1}\left(n_{1}-1\right)}{f^{2}} g_{F}(\nabla f, \nabla f)$ or $r^{B} \leq \mu n_{1}+\frac{n_{1}\left(n_{1}-1\right)}{f^{2}} g_{F}(\nabla f, \nabla f)$, or,

2) either $r^{F} \geq \mu n_{2}+\frac{n_{2}\left(n_{2}-1\right)}{h^{2}} g_{B}(\nabla h, \nabla h)$ or $r^{F} \leq \mu n_{2}+\frac{n_{2}\left(n_{2}-1\right)}{h^{2}} g_{B}(\nabla h, \nabla h)$, then either $\frac{n_{2}}{h} \Delta_{B} h+\frac{n_{1}}{f} \Delta_{F} f \geq 0$ or $\frac{n_{2}}{h} \Delta_{B} h+\frac{n_{1}}{f} \Delta_{F} f \leq 0$.

If $\frac{n_{2}}{h} \Delta_{B} h+\frac{n_{1}}{f} \Delta_{F} f \geq 0$, there are three cases:

(i) $\Delta_{B} h \geq 0$ and $\Delta_{F} f \geq 0$,

(ii) $\Delta_{B} h \leq 0$ and $\Delta_{F} f \geq 0$ with $\frac{n_{2}}{h}\left|\Delta_{B} h\right| \leq \frac{n_{1}}{f}\left|\Delta_{F} f\right|$,

(ii) $\Delta_{B} h \geq 0$ and $\Delta_{F} f \leq 0$ with $\frac{n_{2}}{h}\left|\Delta_{B} h\right| \geq \frac{n_{1}}{f}\left|\Delta_{F} f\right|$.

Therefore $h$ and $f$ become superharmonic or subharmonic function. Since $B$ and $F$ are compact spaces, $h$ and $f$ must be constants.

For $\frac{n_{2}}{h} \Delta_{B} h+\frac{n_{1}}{f} \Delta_{F} f \leq 0$, there are three cases:

(i) $\Delta_{B} h \leq 0$ and $\Delta_{F} f \leq 0$,

(ii) $\Delta_{B} h \leq 0$ and $\Delta_{F} f \geq 0$ with $\frac{n_{2}}{h}\left|\Delta_{B} h\right| \geq \frac{n_{1}}{f}\left|\Delta_{F} f\right|$,

(iii) $\Delta_{B} h \geq 0$ and $\Delta_{F} f \leq 0$ with $\frac{n_{2}}{h}\left|\Delta_{B} h\right| \leq \frac{n_{1}}{f}\left|\Delta_{F} f\right|$.

Therefore $h$ and $f$ are superharmonic or subharmonic function. Since $B$ and $F$ are compact spaces, $h$ and $f$ must be constants.

Thus $M$ is a product semi-Riemannian manifold.

Note: If any of the $\left(B, g_{B}\right)$ and $\left(F, g_{F}\right)$ is compact space, then either $h$ or $f$ will be constant. Therefore, $M$ is a warped product semi-Riemannian manifold.

By above Proposition, we can conclude the following result for the non-existence of connected, compact Einstein doubly warped product manifolds. 
Theorem 3.9. Let $M={ }_{h} B \times{ }_{f} F$ be a connected, compact Einstein DWP semi-Riemannian manifold. Then there does not exist non-trivial connected, compact Einstein DWP semi-Riemannian manifold.

Proof. There will be three situations:

1) If $B$ or $F$ is 1-dimensional manifold. Then it admits a metric with zero scalar curvature.

2) If $B$ or $F$ is 2-dimensional manifold. Then it admits a metric of constant curvature.

3) If $B$ or $F$ is of atleast 3-dimensional manifold. We know that every compact manifold of dimension $\geq 3$ admits a metric of constant negative curvature [20].

Therefore by using the Proposition 3.8 and above three results, we can say that there does not exist non-trivial Einstein doubly warped product manifolds.

Remark 3.10. Doubly warped product Riemannian (semi-Riemannian) manifolds have several applications in theoretical physics. Finsler manifolds are the generalization of Riemannian manifold. Finsler manifold is a manifold $M$, where each tangent space is equipped with a Minkowski norm. This norm also induces a canonical inner product. These Finsler-inner products are not parameterized by points of $M$, but by directions in $T M$. Thus one can think of a Finsler manifold as a space where the inner product does not only depend on where you are, but also in which direction you are looking. Despite this quite large step away from Riemannian geometry, Finsler geometry contains analogues for many of the natural objects in Riemannian geometry. For example, length, geodesics, curvature, connections, covariant derivative, and structure equations all generalize. Presently Finsler geometry has found an abundance of applications in both physics and practical applications.

Warped product (doubly warped) manifold in Finsler setting has been studied by Asanov [2, 3], Kozma et al. [19], Peyghan and Tayebi [25]. Now, it is an open problem that "Does there exist a compact Einstein doubly warped product Finsler manifold with nonconstant warping function?", which is the generalization of Besse [5] open problem.

\section{Acknowledgements}

This work is supported by UGC-BSR Start up Grant Project No. F. 30-12/ 2014. The part of this work is done during the visit of Institute of Mathematics, Polish Academy of Sciences, Poland. 


\section{References}

[1] J. K. Beem, P. Ehrlich and T. G. Powell, Warped product manifolds in relativity Selected Studies: PhysicsAstrophysics, Mathematics, History of Science (Amsterdam: North Holland), 1982, 41-66.

[2] G. S. Asanov, Finslerian extensions of Schwarzschild metric, Fortschr. Phys., 40 (1992), 667-693.

[3] G. S. Asanov, Finslerian metric functions over the product $R \times M$ and their potential applications, Rep. Math. Phys., 41 (1998), 117-132.

[4] J. K. Beem, P. Ehrlich and K. Easley, Global Lorentzian Geometry, 2nd edn, Dekker, New York, 1996.

[5] A. L. Besse, Einstein Manifolds, Springer-Verlag, Berlin-Heidelberg, 1987.

[6] R. L. Bishop and B. O'Neil, Manifolds of negative curvature, Trans. Amer. Math. Soc., 145 (1969), 1-49.

[7] A. S. Diallo, Compact Einstein warped product manifolds, Afr. Mat., 25(2) (2014), 267-270.

[8] F. Dobarro and E. Lami Dozo, Scalar curvatures and warped products of Riemannian geometry, Trans. Amer. Math. Soc., 303 (1987), 161-168.

[9] D. Dumitru, Some aspects concerning compact Einstein warped products, Bull. Transilv. Univ. Braşov Ser. III, 1(50) (2008), 123-127.

[10] K. L. Easely, Local existence of warped product metrics, Ph.D. Thesis, Univ. of Missori-Columbia, 1991.

[11] G. Ganchev and V. Mihoa, Riemannian submanifolds of quasi-constant sectional curvatures, J. Reine Angew. Math., 522 (2000), 119-141.

[12] A. Gebarowski, On Einstein warped products, Tensor (N.S.) 52(3) (1993), 204-207.

[13] A. Gebarowski, Doubly warped products with harmonic Weyl conformal curvature tensor, Colloq. Math., 67 (1994), 73-89.

[14] A. Gebarowski, On conformally recurrent doubly warped products, Tensor (N.S.), 57 (1996), 192-196.

[15] A. N. Hatzinikitas, A note on doubly warped product spaces, arXiv:1403.0204v1.

[16] D.-S. Kim, Compact Einstein warped product spaces, Trends in Math., 5(2) (2002), 1-5.

[17] D.-S. Kim and Y. H. Kim, Compact Einstein warped product spaces with non-positive scalar curvature, Proc. Amer. Math. Soc., 131(8) (2003), 2573-2576.

[18] J.-S. Kim and H.-S. Chung, On Einstein warped products with compact Riemannian base, Honam Math. J., 20(1) (1998), 153-159.

[19] L. Kozma, I. R. Peter and C. Varga, Warped product of Finsler manifolds, Ann. Univ. Sci. Pudapest 44, 157 (2001).

[20] J. Lohkamp, Metrics of negative Ricci curvature, Ann. Math., 140(3) (1994), 655-683.

[21] K. Matsumoto, Doubly warped product manifolds and submanifolds, Global Analysis and Applied Mathematics, AIP Conf. Proc., Amer. Inst. Phys., Melville, 729(2004), 218-224.

[22] M. T. Mustafa, A non-existence result for compact Einstein warped products, J. Phys. A: Math. Gen., 38 (2005), L791-L793.

[23] B. O’Neil, Semi-Riemannian Geometry, Academic Press, New York, 1983.

[24] A. Olteanu, A general inequality for doubly warped product submanifolds, Math. J., Okayama Univ., 52 (2010), 133-142.

[25] E. Peyghan and A. Tayebi, On doubly warped product Finsler manifolds, Nonlinear Analysis: Real World Appl., 13(4) (2012), 1703-1720.

[26] S. Sular and C. Özg̈ur, Doubly warped product submanifolds of $(\kappa, \mu)$-contact metric manifolds, Ann. Polon. Math., 100 (2011), 223-236.

[27] B. Ünal, Doubly warped products, Diff. Geom. Appl., 15(3) (2001), 253-263.

Department of Mathematics \& Statistics, School of Mathematical \& Physical Sciences, Dr. Harisingh Gour University, Sagar-470003, Madhya Pradesh, India.

E-mail: punam_2101@yahoo.co.in 